J. Environ. Sci.

Institute of Environmental Studies and Research - Ain Shams University

\title{
QUALITY ASSESSMENT OF RAISING GROUNDWATER IN WATER LOGGING AREAS IN ASWAN CITY
}

\author{
Mohamed, A. Afifi ${ }^{(1)}$; Taha. M. Abdel $\operatorname{Razek}^{(2)}$ \\ and Refat. Sh. Abdel Wahab ${ }^{(3)}$
}

1) Research and Development sector, Holding Company for Water and Wastewater 2) Department of Environmental Basic Sciences, Institute of Environment Studies and Research 3) Environmental Science Division, National Research Center

\begin{abstract}
Aswan is considered one of the important Egyptian Governorates as it represents the southern gate to Egypt. Moreover, the existences of Aswan High Dam which represent a strategic element of the Egyptian country give this city high importance. The present study deals with the quality assessing the rising groundwater the aquifer into the logged areas in Aswan city using Water Quality Index approach and gives a recommendation for using water for drinking. Eight water samples are collected between December 2016 to June 2017 Physio-chemical parameter are estimated and processed according to Standard Methods for the Examination of Water and Wastewater (21st edition - SMWW). According to the international standards of assessing water quality for drinking and irrigation uses and national laws and rules for using and discharge of groundwater and drainage water into the River Nile or irrigation Canal. TDS in the rising groundwater is relatively high; it ranged from 235 to $6794 \mathrm{mg} / \mathrm{l}$. It also has a highly polluting effect on water quality. It has nitrate concentration ranges from 0.2,82 to $204.59 \mathrm{mg} / \mathrm{l}$. Samples of Alnubalaa graveyard, Kima leakage, and Leakage behind Spanish bazar samples show characters showed WQI of 71.2, 79.1, and 57.2 respectively, that resemble to be river Nile water or irrigated water and cannot be used for drinking. Groundwater sample was collected from Alshallal well has 86.9 WQI. It can be used as drinking water after some disinfection treatment with chlorine. other sites have WQI (drinking water- raw water) \{ Al Hasaya House (23.8-31.1), Aldamas Leakage House (38.5-45), Alsail House (16-27.1) and Khor awada House (24.3-26) \} have WQI for (drinking water- raw water),


show bad criteria compared to drinking water standards and raw water standards and can be recommended doing some treatment before discharging to River Nile or using in irrigation.

Key words: and Phrases: Water Quality, groundwater, Water Quality Index, drinking water, River Nile, raised groundwater in logged areas, deep well.

\section{INTRODUCTION}

Environmental pollution problems are one of the most serious national problems which require great efforts at all levels; either, national and international. This is especially true with respect to pollution of rivers because they serve as the recipient of urban and rural wastewater. Water quality issues have become of major concern to all agencies dealing with water resources management and planning. This requires data collection, analysis, and interpretation. One major goal of surface water quality data collection may be the estimation of the magnitude of changes in the concentration of various constituents (Yehia and Sabae., 2011).

Human activities mainly impact surface water and groundwater quality through effluent discharges, using agricultural chemicals, in addition to the increased exploitation of water resources. Many rivers in the developing countries are heavily polluted due to anthropogenic activities (Jonnalagadda and Mgere 2011).

Over the last few years, a steady rise in groundwater levels has been observed in several parts of Aswan City. This reflect environmental problems existing in many areas of the city, where it creates swamps, logged areas and ponds and affects the foundation of many buildings as it appears in El-Seil, Khor Awada, Phantomic graves, El-Aqad buildings, Blood Bank, Military building, El Shallal and KIMA Factory area. Rising groundwater levels in 2 
water logged areas are expected to be a chronic problem and will likely be a major issue for residential areas of Aswan city (Selim et al., 2014).

Water quality index (WQI) is considered as one of the most effective tools which can be used to evaluate the water quality status for drinking using a single value indicating the overall quality of water that describes the status of water quality to the public as well as decision and policy makers. It is based on the ensemble of the records of physical, chemical and biological parameters of the water body using specific methods (Elshemy et al., 2016).

\section{The present study deals with the following:}

1. The status of the rising groundwater in logged areas resources in Aswan city.

2. Assessing the quality of the groundwater for drinking in Aswan City using Water Quality Index approach.

3. Providing a recommendation for the treatment of water resources environmental pollution.

\section{MATERIAL AND METHODS}

Water quality assessment is carried out through sampling 8 water samples collected three times between December 2016 and June 2017 and running physiochemical analyses. These samples are distributed along the study area and representing different water types (Rising groundwater in logged areas and Deep groundwater in wells. Rising groundwater (Samples in logged areas) samples were collected from 8 sites (Figure1). These sites are Al Hasaya House, Alsail House, Khor awada House, Berket Aldamas 
Leakage House, Alnubalaa graveyard, Kima Leakage, Well 5 Alshallal water plant and Leakage behind Spanish bazar.

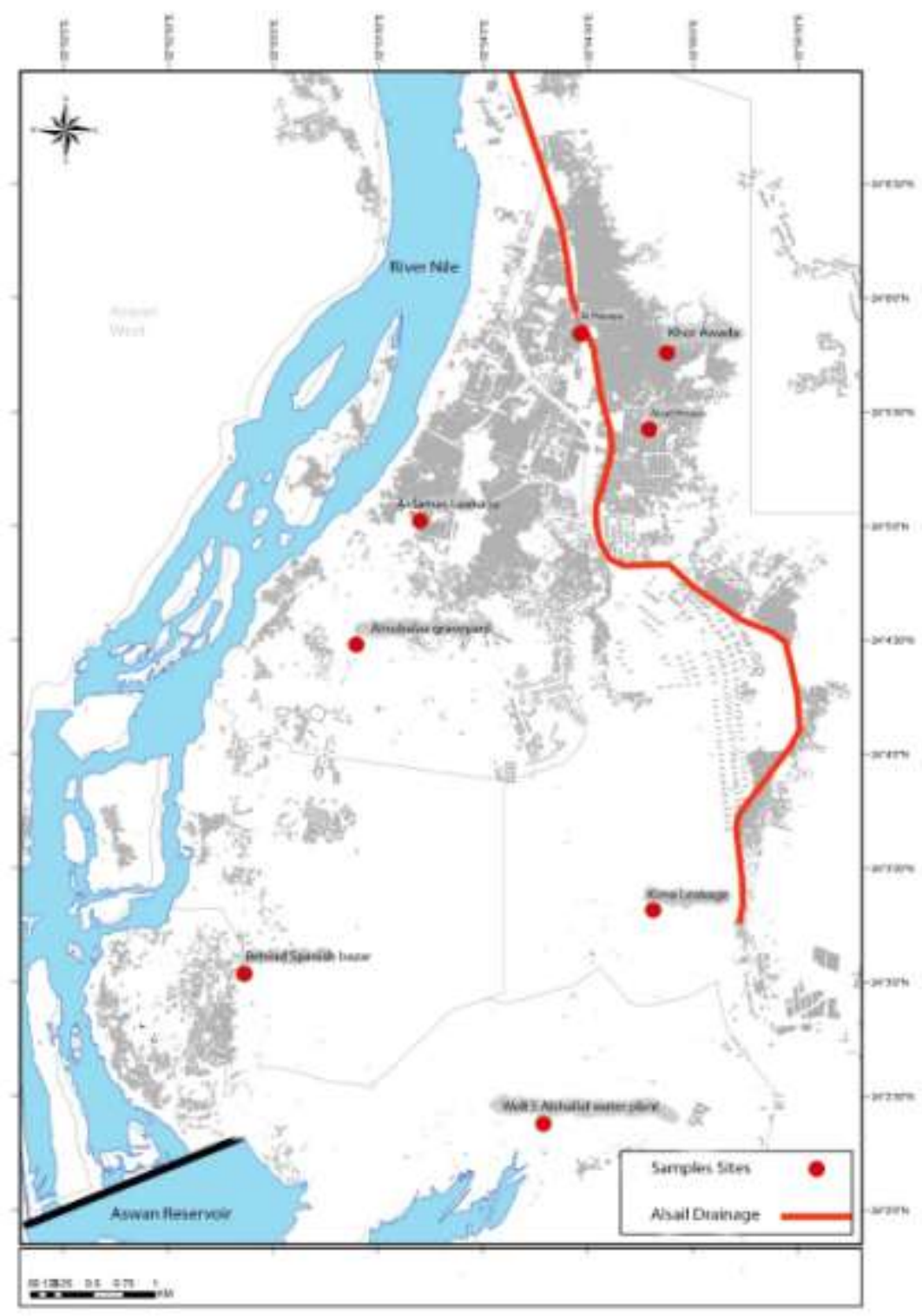

Figure (1): sampling points and water logging areas 
Sampling and onsite field measurements are done according to the international standards. Eight samples are collected from waterlogged areas (rising groundwater). Before measuring the field parameters for water quality (pH, E.C., Temp, $\mathrm{O} 2$ and residual chlorine) the site is pumped subjected to pumping to reach the steady state for measurements of EC and temperature.

Samples are collected and analysed according to Standard Methods for the Examination of Water and Wastewater 21st edition - SMWW. (Eaton et $a l, 1998)$ As well as the Canadian Water Quality Index (WQI) from Canadian Council of Ministers of the Environment (CCME) by using equation to indicate water quality according to several standards including drinking water standard, aquifers standard and river raw water standard. (CCME, 2017)

WQI Calculation of the CCME: The water quality index (WQI) were developed by the CCME and employs the combination of three essential measures of variance such as scope, frequency, and amplitude. Detailed calculation procedures and classification are explained in Canadian Water Quality Index 1.0 - Technical report as follows [10].

Scope $\left(F_{1}\right)$ represents the extent of water quality guideline noncompliance over the time period of interest. F1 is expressed as indicated in Equation 01.

$$
F_{1}=\frac{\text { Number of failed variables }}{\text { Total number of variables }} \times 100 \quad \text { Equation } 01
$$

Frequency $\left(\mathrm{F}_{2}\right)$ represents the percentage of individual tests that do not meet the guidelines called "failed tests" as expressed in Equation 02.
$F_{2}=\frac{\text { Number of failed tests }}{\text { Total number of tests }} \times 100$
Equation 02 
Amplitude $\left(\mathrm{F}_{3}\right)$ represents the amount by which failed test values do not meet their guidelines. Amplitude is calculated in three steps.

i. The number of times by which an individual concentration is greater than (or less than, when the guideline is a minimum) the objective is termed an "excursion" and is expressed as follows. When the test value must not exceed the objective (Equation 03)

excursion $_{i}=\frac{\text { Failed test value } i}{\text { objective } / \text { guideline } i}-1 \quad$ Equation 03

For the cases in which the test value must not fall below the objective (Equation 04)

excursioni $=\frac{\text { objective } / \text { guideline } i}{\text { Failed test value } i}-1 \quad$ Equation 04

ii. The collective amount by which individual tests are out of compliance is calculated by summing the excursions of individual tests from their objectives and dividing by the total number of tests (both those meeting objectives and those not meeting objectives). This variable referred to as the normalized sum of excursions, (nse) is calculated as Equation 05.

$n s e=\frac{\sum_{i=1}^{n} \text { excursion } i}{\text { Number of tests }} \quad$ Equation 05

iii. The amplitude F3 is calculated by an asymptotic function that scales the normalized sum of the excursions from objectives (nse) to yield a range between 0 and 100. Mathematically, F3 is expressed as indicated in Equation 06.

$\begin{array}{ll}F_{3}=\frac{n s e}{0.01 n s e+0.01} & \text { Equation } 06\end{array}$ 


\section{The CCME WQI is then calculated as,}

CCME WQI $=100-\frac{\sqrt{f 1^{2}+f 2^{2}+f 3^{2}}}{1.732}$

Equation 07

CCME WQI value has been determined, water quality can be ranked by relating it to one of the following categories

\begin{tabular}{|c|c|c|c|c|}
\hline \multicolumn{5}{|c|}{ CCME WQI guide line } \\
\hline Poor & Marginal & Fair & Good & Excellent \\
\hline $\begin{array}{c}\text { (CCME } \\
\text { WQI Value } \\
0-44)- \\
\text { water } \\
\text { quality is } \\
\text { almost } \\
\text { always } \\
\text { threatened } \\
\text { or impaired; } \\
\text { conditions } \\
\text { usually } \\
\text { depart from } \\
\text { natural or } \\
\text { desirable } \\
\text { levels. }\end{array}$ & $\begin{array}{l}\text { (CCME WQI } \\
\text { Value 45-64) } \\
\text { - water } \\
\text { quality is } \\
\text { frequently } \\
\text { threatened or } \\
\text { impaired; } \\
\text { conditions } \\
\text { often depart } \\
\text { from natural } \\
\text { or desirable } \\
\text { levels. }\end{array}$ & $\begin{array}{c}\text { (CCME WQI } \\
\text { Value 65-79) } \\
\text { - water } \\
\text { quality is } \\
\text { usually } \\
\text { protected but } \\
\text { occasionally } \\
\text { threatened or } \\
\text { impaired; } \\
\text { conditions } \\
\text { sometimes } \\
\text { depart from } \\
\text { natural or } \\
\text { desirable } \\
\text { levels. }\end{array}$ & $\begin{array}{c}\text { (CCME } \\
\text { WQI Value } \\
\text { 80-94)- } \\
\text { water } \\
\text { quality is } \\
\text { protected } \\
\text { with only a } \\
\text { minor } \\
\text { degree of } \\
\text { threat or } \\
\text { impairment; } \\
\text { conditions } \\
\text { rarely } \\
\text { depart from } \\
\text { natural or } \\
\text { desirable } \\
\text { levels. }\end{array}$ & $\begin{array}{l}\text { (CCME WQI } \\
\text { Value 95-100)- } \\
\text { water quality is } \\
\text { protected with a } \\
\text { virtual absence } \\
\text { of threat or } \\
\text { impairment; } \\
\text { conditions very } \\
\text { close to natural } \\
\text { or pristine } \\
\text { levels. These } \\
\text { index values can } \\
\text { only be obtained } \\
\text { if all } \\
\text { measurements } \\
\text { are within } \\
\text { objectives } \\
\text { virtually all of } \\
\text { the time. }\end{array}$ \\
\hline
\end{tabular}

\section{RESULTS}

Results for water quality analyses including both physico-chemical and microbiological parameters are shown in Tables (1,2 and 3) during the period from December 2016 to June 2017. There is a significant difference between water quality of rising groundwater in the logged areas and that from deep 
groundwater (wells) which has fresh groundwater with a TDS average 258 $\mathrm{mg} / \mathrm{l}$. The water TDS in the leakage is relatively high; it ranged from 235 to $6794 \mathrm{mg} / \mathrm{l}$. It is highly polluted; the nitrate concentration ranges from 0.2.82 to $204.59 \mathrm{mg} / \mathrm{l}$.

Table (1): Physico-chemical and microbiological characteristics of rising groundwater samples in some area of Aswan city

\begin{tabular}{|c|c|c|c|c|c|c|c|c|c|}
\hline \multirow{2}{*}{\multicolumn{2}{|c|}{ 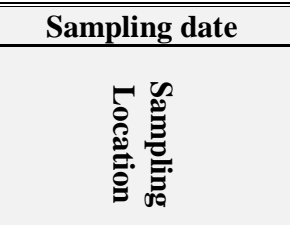 }} & \multicolumn{8}{|c|}{$12 / 2016$} \\
\hline & & 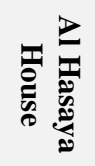 & 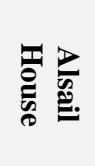 & 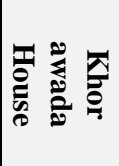 & 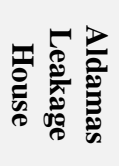 & 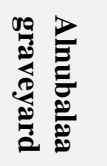 & 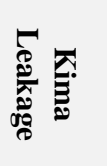 & 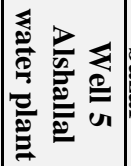 & 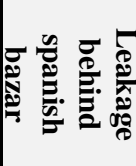 \\
\hline Parameter & Unit & & & & & & & & \\
\hline $\mathrm{pH}$ & - & 8.4 & 8.1 & 8.1 & 8.5 & 7.8 & 6.9 & 7.1 & 6.4 \\
\hline $\begin{array}{c}\text { Electrical } \\
\text { Conductivity }\end{array}$ & $\mu \mathrm{S} / \mathrm{cm}$ & 10020 & 2690 & 3700 & 1094 & 344 & 557 & 434 & 1108 \\
\hline TDS & $\mathrm{mg} / \mathrm{L}$ & 6015 & 1610 & 2220 & 660 & 210 & 335 & 262 & 665 \\
\hline T. Hardness & $\mathrm{mg} / \mathrm{L}$ & 260 & 483 & 235 & 91 & 100 & 132 & 157 & 71 \\
\hline Sodium & $\mathrm{mg} / \mathrm{L}$ & 2196 & 340 & 701 & 187 & 27 & 53 & 20 & 231 \\
\hline Potassium & $\mathrm{mg} / \mathrm{L}$ & 85 & 23 & 19 & 33 & 4 & 6 & 4 & 2 \\
\hline Chloride & $\mathrm{mg} / \mathrm{L}$ & 1511 & 425 & 531 & 75 & 11 & 33 & 7 & 48 \\
\hline Sulfate & $\mathrm{mg} / \mathrm{L}$ & 3340 & 487 & 860 & 121 & 19 & 43 & 15 & 117 \\
\hline Iron & $\mathrm{mg} / \mathrm{L}$ & 0.145 & 0.325 & $<0.05$ & 0.075 & 0.218 & $<0.05$ & 0.064 & 0.355 \\
\hline Manganese & $\mathrm{mg} / \mathrm{L}$ & $<0.025$ & $<0.025$ & $<0.025$ & $<0.025$ & $<0.025$ & 0.042 & 0.107 & $<0.025$ \\
\hline Nitrate & $\mathrm{mg} / \mathrm{L}$ & 2.8 & 187.1 & 168.9 & 24.7 & 4.2 & 2.5 & 0.73 & 0.71 \\
\hline Nitrite & $\mathrm{mg} / \mathrm{L}$ & ND & 0.29 & 0.29 & 0.1 & 0.07 & 0.07 & 0.15 & 0.06 \\
\hline Fluoride & $\mathrm{mg} / \mathrm{L}$ & 0.83 & 0.35 & 0.62 & 0.59 & 0.46 & 0.4 & 0.32 & 3 \\
\hline Ammonia & $\mathrm{mg} / \mathrm{L}$ & 0.61 & 0.83 & 0.88 & 0.61 & 0.53 & 0.58 & 0.69 & ND \\
\hline $\begin{array}{c}\text { Total } \\
\text { Bacterial } \\
\text { Counts }\end{array}$ & $\begin{array}{c}\mathrm{CFU} / \\
\mathrm{mL}\end{array}$ & 11540 & 25080 & 13250 & 26790 & 6560 & 25370 & 40 & 27220 \\
\hline
\end{tabular}


J. Environ. Sci.

Institute of Environmental Studies and Research - Ain Shams University

Table (2): Physico-chemical and microbiological characteristics of rising groundwater samples in some area of Aswan city

\begin{tabular}{|c|c|c|c|c|c|c|c|c|c|}
\hline \multirow{2}{*}{\multicolumn{2}{|c|}{ 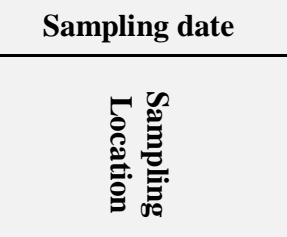 }} & \multicolumn{8}{|c|}{ 03/2017 } \\
\hline & & \multirow[t]{2}{*}{ 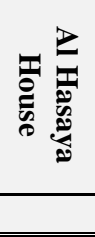 } & \multirow[t]{2}{*}{ 要㿣 } & \multirow[t]{2}{*}{ 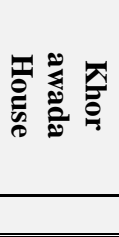 } & \multirow[t]{2}{*}{ 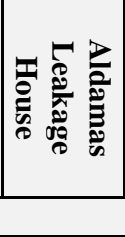 } & \multirow[t]{2}{*}{ 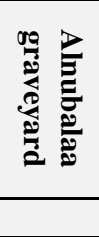 } & \multirow[t]{2}{*}{ 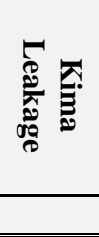 } & \multirow[t]{2}{*}{ 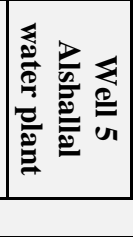 } & \multirow{2}{*}{ 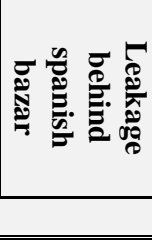 } \\
\hline Parameter & Unit & & & & & & & & \\
\hline $\mathrm{pH}$ & - & 8 & 8.1 & 8.2 & 8.4 & 7.3 & 7 & 7.2 & 6.3 \\
\hline $\begin{array}{c}\text { Electrical } \\
\text { Conductivity }\end{array}$ & $\mu \mathrm{S} / \mathrm{cm}$ & 11301 & 3040 & 4181 & 1236 & 389 & 629 & 422 & 1252 \\
\hline TDS & $\mathrm{mg} / \mathrm{L}$ & 6781 & 1824 & 2509 & 742 & 233 & 378 & 253 & 751 \\
\hline T. Hardness & $\mathrm{mg} / \mathrm{L}$ & 286 & 546 & 261 & 103 & 114 & 139 & 155 & 79 \\
\hline Sodium & $\mathrm{mg} / \mathrm{L}$ & 2471 & 376 & 784 & 211 & 33 & 57 & 20 & 259 \\
\hline Potassium & $\mathrm{mg} / \mathrm{L}$ & 91 & 27 & 22 & 37 & 6 & 8 & 5 & 51 \\
\hline Chloride & $\mathrm{mg} / \mathrm{L}$ & 1705 & 481 & 605 & 85 & 12 & 35 & 8 & 55 \\
\hline Sulfate & $\mathrm{mg} / \mathrm{L}$ & 3770 & 541 & 981 & 137 & 23 & 49 & 14 & 134 \\
\hline Iron & $\mathrm{mg} / \mathrm{L}$ & 0.164 & 0.690 & $<0.05$ & 0.085 & 0.239 & $<0.05$ & 0.061 & 0.405 \\
\hline Manganese & $\mathrm{mg} / \mathrm{L}$ & $<0.025$ & $<0.025$ & $<0.025$ & $<0.025$ & $<0.025$ & 0.06 & 0.11 & $<0.025$ \\
\hline Nitrate & $\mathrm{mg} / \mathrm{L}$ & 3.13 & 201.36 & 188.92 & 27.91 & 4.61 & 2.81 & 0.71 & 0.81 \\
\hline Nitrite & $\mathrm{mg} / \mathrm{L}$ & ND & 0.31 & 0.31 & 0.11 & 0.07 & 0.09 & 0.16 & 0.08 \\
\hline Fluoride & $\mathrm{mg} / \mathrm{L}$ & 0.91 & 0.39 & 0.68 & 0.67 & 0.50 & 0.43 & 0.33 & 3.41 \\
\hline Ammonia & $\mathrm{mg} / \mathrm{L}$ & 0.66 & 0.88 & 0.91 & 0.69 & 0.59 & 0.61 & 0.68 & ND \\
\hline $\begin{array}{c}\text { Total } \\
\text { Bacterial } \\
\text { Counts }\end{array}$ & $\begin{array}{c}\mathrm{CFU} / \\
\mathrm{mL}\end{array}$ & 11430 & 24320 & 12980 & 27450 & 6140 & 26510 & 33 & 27670 \\
\hline
\end{tabular}


Table (3): Physico-chemical and microbiological characteristics of rising groundwater samples in some area of Aswan city

\begin{tabular}{|c|c|c|c|c|c|c|c|c|c|}
\hline \multirow{2}{*}{\multicolumn{2}{|c|}{ 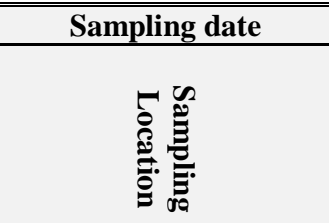 }} & \multicolumn{8}{|c|}{$06 / 2017$} \\
\hline & & \multirow[t]{2}{*}{ 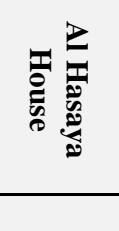 } & \multirow[t]{2}{*}{ 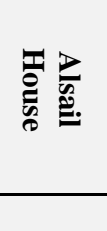 } & \multirow[t]{2}{*}{ 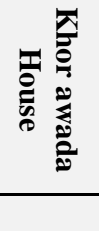 } & \multirow[t]{2}{*}{ 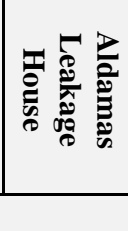 } & \multirow[t]{2}{*}{ 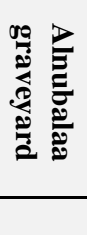 } & \multirow[t]{2}{*}{ অ: } & \multirow[t]{2}{*}{ 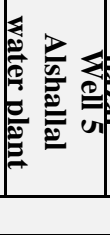 } & \multirow[t]{2}{*}{ 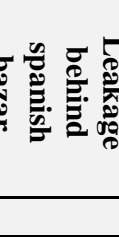 } \\
\hline Parameter & Unit & & & & & & & & \\
\hline $\mathrm{pH}$ & - & 8.1 & 8 & 8.1 & 8.4 & $\overline{7.5}$ & $\overline{77.1}$ & 7 & 46.2 \\
\hline $\begin{array}{c}\text { Electrical } \\
\text { Conductivity }\end{array}$ & $\mu \mathrm{S} / \mathrm{cm}$ & 12645.82 & 3401.42 & 4678.54 & 1383.33 & 434.98 & 704.31 & 429.0 & 1401.03 \\
\hline TDS & $\mathrm{mg} / \mathrm{L}$ & 7587 & 2041 & 2807 & 830 & 261 & 423 & 257 & 841 \\
\hline T. Hardness & $\mathrm{mg} / \mathrm{L}$ & 320 & 611 & 292 & 115 & 128 & 156 & 154 & 88 \\
\hline Sodium & $\mathrm{mg} / \mathrm{L}$ & 2765 & 421 & 877 & 236 & 37 & 64 & 21 & 290 \\
\hline Potassium & $\mathrm{mg} / \mathrm{L}$ & 102 & 30 & 25 & 42 & 7 & 9 & 4 & 57 \\
\hline Chloride & $\mathrm{mg} / \mathrm{L}$ & 1908 & 538 & 677 & 95 & 13 & 39 & 7 & 62 \\
\hline Sulfate & $\mathrm{mg} / \mathrm{L}$ & 4219 & 605 & 1098 & 153 & 26 & 54 & 16 & 150 \\
\hline Iron & $\mathrm{mg} / \mathrm{L}$ & 0.18 & 0.77 & $<0.05$ & 0.09 & 0.27 & $<0.05$ & 0.07 & 0.45 \\
\hline Manganese & $\mathrm{mg} / \mathrm{L}$ & $<0.025$ & $<0.025$ & $<0.025$ & $<0.025$ & $<0.025$ & 0.07 & 0.13 & $<0.025$ \\
\hline Nitrate & $\mathrm{mg} / \mathrm{L}$ & 3.50 & 225.32 & 211.40 & 31.23 & 5.16 & 3.14 & 0.74 & 0.91 \\
\hline Nitrite & $\mathrm{mg} / \mathrm{L}$ & ND & 0.35 & 0.35 & 0.13 & 0.08 & 0.10 & 0.17 & 0.09 \\
\hline Fluoride & $\mathrm{mg} / \mathrm{L}$ & 1.02 & 0.44 & 0.76 & 0.75 & 0.56 & 0.48 & 0.35 & 3.82 \\
\hline Ammonia & $\mathrm{mg} / \mathrm{L}$ & 0.74 & 0.98 & 1.02 & 0.77 & 0.66 & 0.68 & 0.76 & ND \\
\hline $\begin{array}{c}\text { Total } \\
\text { Bacterial } \\
\text { Counts }\end{array}$ & $\mathrm{CFU} / \mathrm{mL}$ & 12790 & 27214 & 14525 & 30717 & 6871 & 29665 & 37 & 30963 \\
\hline
\end{tabular}


J. Environ. Sci.

Institute of Environmental Studies and Research - Ain Shams University

Table (4): Physico-chemical and microbiological characteristics of rising groundwater samples in water logged areas in Aswan city (Average of three samples $\pm \mathrm{SD}$ ) $\mathrm{SD}=$ Standard Deviation

\begin{tabular}{|c|c|c|c|c|c|c|c|c|c|}
\hline \multicolumn{2}{|c|}{ 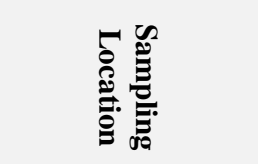 } & 赵 & 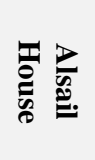 & 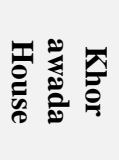 & 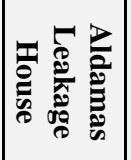 & 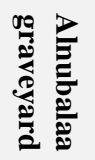 & 可 & 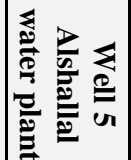 & 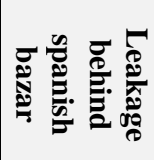 \\
\hline Parameter & Unit & & & & & & & & \\
\hline $\mathrm{pH}$ & - & 8.2 & 8.1 & 8.1 & 8.4 & 7.5 & 7.0 & 7.1 & 6.3 \\
\hline E.C. & $\begin{array}{c}\mu \mathrm{S} / \mathrm{c} \\
\mathrm{m}\end{array}$ & 11322 & 3044 & 4187 & 1238 & 389 & 630 & 428 & 1254 \\
\hline TDS & $\mathrm{mg} / \mathrm{L}$ & 6794 & 1825 & 2512 & 744 & 235 & 378 & 258 & 752 \\
\hline $\begin{array}{c}\mathrm{T} . \\
\text { Hardness }\end{array}$ & $\mathrm{mg} / \mathrm{L}$ & 289 & 547 & 263 & 103 & 114 & 142 & 155 & 79 \\
\hline Sodium & $\mathrm{mg} / \mathrm{L}$ & 2477 & 379 & 787 & 212 & 32 & 58 & 20 & 260 \\
\hline Potassium & $\mathrm{mg} / \mathrm{L}$ & 93 & 27 & 22 & 37 & 6 & 8 & 4 & 37 \\
\hline Chloride & $\mathrm{mg} / \mathrm{L}$ & 1708 & 481 & 604 & 85 & 12 & 36 & 7 & 55 \\
\hline Sulfate & $\mathrm{mg} / \mathrm{L}$ & 3776 & 544 & 980 & 137 & 23 & 49 & 15 & 134 \\
\hline Iron & $\mathrm{mg} / \mathrm{L}$ & 0.164 & 0.596 & $<0.05$ & 0.085 & 0.241 & $<0.05$ & 0.064 & 0.404 \\
\hline Manganese & $\mathrm{mg} / \mathrm{L}$ & $<0.025$ & $<0.025$ & $<0.025$ & $<0.025$ & $<0.025$ & 0.06 & 0.11 & $<0.025$ \\
\hline Nitrate & $\mathrm{mg} / \mathrm{L}$ & 3.14 & 204.59 & 189.74 & 27.95 & 4.66 & 2.82 & 0.73 & 0.81 \\
\hline Nitrite & $\mathrm{mg} / \mathrm{L}$ & ND & 0.315 & 0.316 & 0.113 & 0.073 & 0.087 & 0.16 & 0.077 \\
\hline Fluoride & $\mathrm{mg} / \mathrm{L}$ & 0.92 & 0.39 & 0.69 & 0.67 & 0.51 & 0.44 & 0.33 & 3.41 \\
\hline Ammonia & $\mathrm{mg} / \mathrm{L}$ & 0.67 & 0.90 & 0.94 & 0.69 & 0.59 & 0.62 & 0.71 & ND \\
\hline $\begin{array}{c}\text { Total } \\
\text { Bacterial } \\
\text { Counts }\end{array}$ & $\begin{array}{c}\mathrm{CFU} / \\
\mathrm{mL}\end{array}$ & 11920 & 25538 & 13585 & 28319 & 6524 & 27182 & 37 & 28618 \\
\hline
\end{tabular}


Table (5): Statistical Calculation of parameters (in ppm)

\begin{tabular}{|c|c|c|c|c|c||}
\hline & Minimum & Maximum & Mean & Median & STD \\
\hline \hline $\mathrm{pH}$ & 6.3 & 8.4 & 7.6 & 7.8 & 0.739 \\
\hline $\begin{array}{c}\text { Electrical } \\
\text { Conductivity }\end{array}$ & 389.2 & 11322.3 & 2811.5 & 1245.8 & 3695.282 \\
\hline TDS & 234.7 & 6794.4 & 1687.3 & 748.1 & 2217.225 \\
\hline T. Hardness & 79.5 & 546.5 & 211.5 & 148.8 & 154.695 \\
\hline Sodium & 20.3 & 2477.3 & 528.2 & 235.8 & 826.859 \\
\hline Potassium & 4.333 & 92.610 & 29.101 & 24.305 & 28.893 \\
\hline Chloride & 7.333 & 1708.0 & 373.6 & 69.9 & 586.677 \\
\hline Sulfate & 14.9 & 3776.2 & 707.1 & 135.3 & 1284.822 \\
\hline Iron & 0.064 & 0.596 & 0.259 & 0.203 & 0.206 \\
\hline Manganese & 0.056 & 0.114 & 0.085 & 0.085 & 0.041 \\
\hline Nitrate & 0.727 & 204.594 & 54.305 & 3.900 & 88.713 \\
\hline Nitrite & 0.073 & 0.316 & 0.163 & 0.113 & 0.108 \\
\hline Fluoride & 0.333 & 3.409 & 0.919 & 0.587 & 1.024 \\
\hline Ammonia & 0.593 & 0.936 & 0.732 & 0.690 & 0.133 \\
\hline $\begin{array}{c}\text { Total Bacterial } \\
\text { Counts }\end{array}$ & 37 & 28618 & 17715 & 19561 & 11152 \\
\hline \hline
\end{tabular}

Table (6): WQI Data Summary according to drinking water guidelines

\begin{tabular}{|c|c|c|c|}
\hline Station & Index Period & CCME WQI & WQI Category \\
\hline \hline Al Hasaya House & $2016-2017$ & 23.8 & POOR \\
\hline Aldamas Leakage House & $2016-2017$ & 38.5 & POOR \\
\hline Alnubalaa graveyard & $2016-2017$ & 43.9 & POOR \\
\hline Alsail House & $2016-2017$ & 16 & POOR \\
\hline Khor awada House & $2016-2017$ & 24.3 & POOR \\
\hline Kima Leakage & $2016-2017$ & 40.5 & POOR \\
\hline Leakage behind spanish bazar & $2016-2017$ & 31.1 & POOR \\
\hline Well 5 Alshallal water plant & $2016-2017$ & 86.9 & Good \\
\hline
\end{tabular}


J. Environ. Sci.

Institute of Environmental Studies and Research - Ain Shams University

Figer 1: WQI Data Summary according to drinking water guidelines

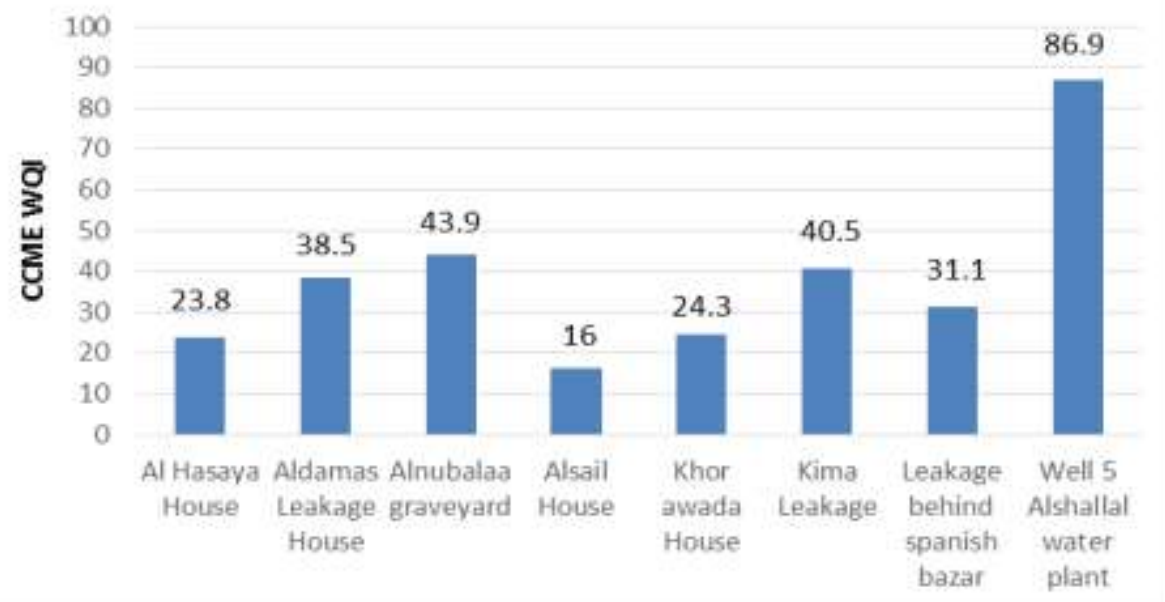

From the water quality index calculation in (Table 3 and Figure 1) all water in the logged areasare poor to drinking water criteria all raised location (Al Hasaya House, Aldamas Leakage House, Alnubalaa graveyard, Alsail House, Khor awada House, Kima Leakage, Leakage behind and Spanish bazar failed in nitrate, ammonia and total bacterial count but the sample from well 5 groundwater show good criteria to drinking water.

Table (7): WQI Data Summary according to raw water guidelines

\begin{tabular}{|c|c|c|c|}
\hline Station & Index Period & CCME WQI & WQI Category \\
\hline \hline Al Hasaya House & $2016-2017$ & 31.1 & POOR \\
\hline Aldamas Leakage House & $2016-2017$ & 45 & MARGINAL \\
\hline Alnubalaa graveyard & $2016-2017$ & 71.2 & FAIR \\
\hline Alsail House & $2016-2017$ & 27.1 & POOR \\
\hline Khor awada House & $2016-2017$ & 26 & POOR \\
\hline Kima Leakage & $2016-2017$ & 79.1 & FAIR \\
\hline Leakage behind spanish bazar & $2016-2017$ & 57.2 & MARGINAL \\
\hline Well 5 Alshallal water plant & $2016-2017$ & 89.4 & GOOD \\
\hline
\end{tabular}




\section{Figer 3: WQI Data Summary according to raw water guidelines}

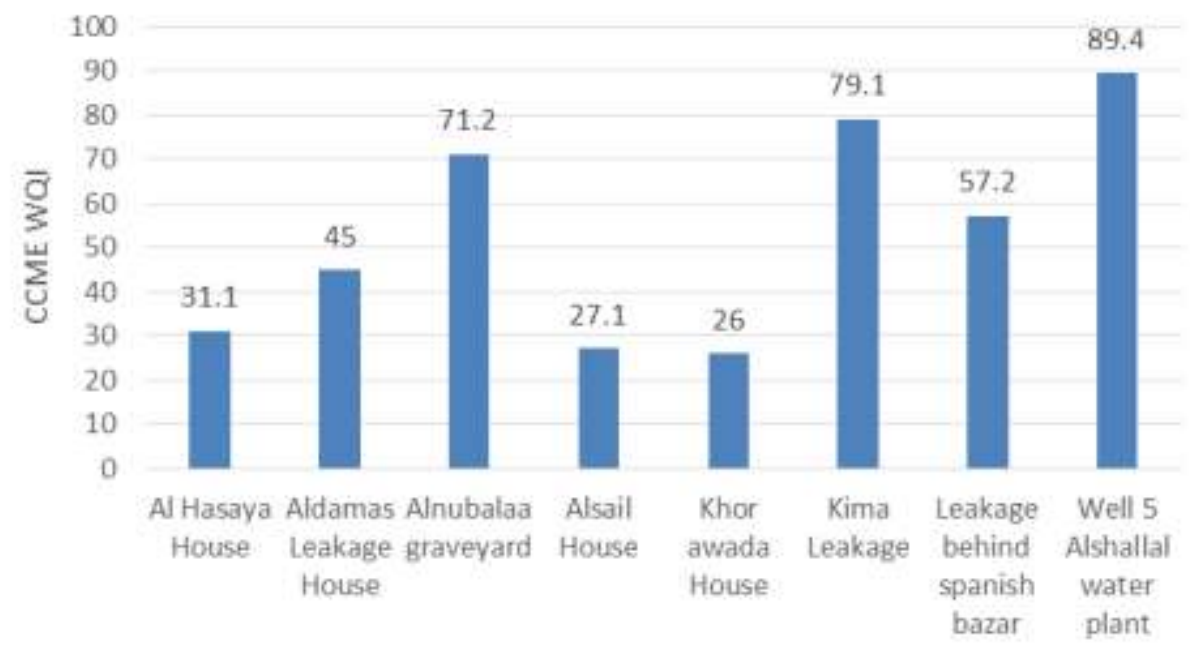

Depending on raw water criteria and data from WQI calculation (table 4) it was found that Alnubalaa graveyard, Kima leakage and well 5 in Alshallal water plant are good for discharge into the river or use in irrigation. Leakage behind Spanish bazar is marginal because it is low in $\mathrm{pH}$ and has value in TDS and fluoride exceeds the standard value.

\section{CONCLUSION}

8 Water samples are collected from the different location, in logged areas in Aswan City three times between Decembers 2016 and June 2017 they are assesssed, using Canadian Water Quality Index (CCME) equations to indicate water quality according to drinking water standard and river raw water standard. The water samples were collected from houses Alnubalaa graveyard, Kima leakage, and Leakage behind Spanish bazar have chemical and bacteriological characters near to be river Nile water but it cannot use for drinking. 
According to the international standards of assessing water quality for drinking and irrigation uses and national laws and rules for using and discharge of groundwater and drainage water into the River Nile or irrigation Canal.

The Water samples collected from groundwater in the quantity aquifers can be used for drinking water after some disinfection treatment with chlorine.

Other sites of logged areas such as Al Hasaya House, Aldamas Leakage House Alsail House, and Khor awada House the collected water samples bad criteria comparing to drinking water standard and raw water standers and they need for treatment before discharging it to River Nile or using for irrigation.

\section{REFERENCES}

Eaton, A. D.; Clesceri, L. S.; Greenberg, A. E. and Franson, M. A. H. (1998): Standard methods for the examination of water and wastewater. Washington, DC, American Public Health Association.

Elshemy, Zeidan and Hassan (2016): Water Quality Assessment of Rosetta Branch, Nile River, Egypt. Third International Environmental Forum, Environmental Pollution: Problem \& Solution, Tanta University, Egypt, July $12^{\text {th }}-14^{\text {th }}, 2016$.

Jonnalagadda, S. B. and Mgere, G.: Water quality of the Odzi River in the eastern high lands of Zimbabwe. Water Res. 2001; 35(10): 23712376.

Niemi, G. J.; Devore, P.; Detenbeck, N.; Taylor, D. and Lima, A (1990): Overview of case studies on recovery of aquatic systems from disturbance. Environmental management. 14: 571-87. 
Selim, S. A.; Hamdan, A. M. and Rady, A. A. (2014): Groundwater Rising as Environmental Problem, Causes and Solutions: Case Study from Aswan City, Upper Egypt. Open Journal of Geology, 4, 324-341.

Yehia, H. and S. Sabae, (2011): Microbial Pollution of Water in El-Salam Canal, Egypt. American-Eurasian Journal of Agricultural \& Environmental Science, 11(2): 305-309.

Canadian Council of Ministers of the Environment CCME (2017): Alberta, British Columbia, Canada, Manitoba, New Brunswick, Newfoundland and Labrador, Northwest Territories, Nova Scotia, Nunavut, Ontario, Prince Edward Island, Québec, Saskatchewan and Yukon, p. https://www.ccme.ca. Available at: https://www.ccme.ca/files/Resources/water/water_quality/WQI.zi $\mathrm{p}$

قانون رقم ^^ لسنة r1911 للصرف على الخزانات الجوفية وفروع وروافد النيل والمجري الرئيسي لنهر النيل والمصارف البلدية والصنعية.

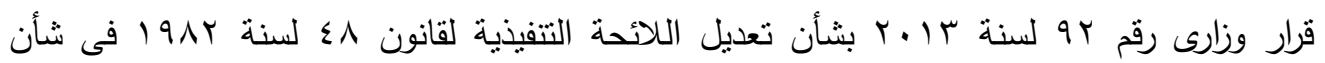
حماية نهر النيل والمجارى المائية

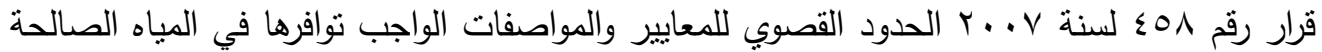
لمثرب والاستخدام المنزلي 


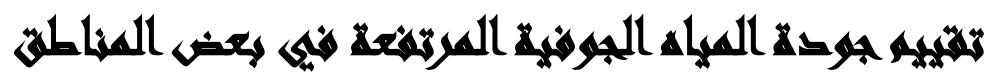

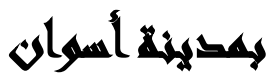

\section{[1]}

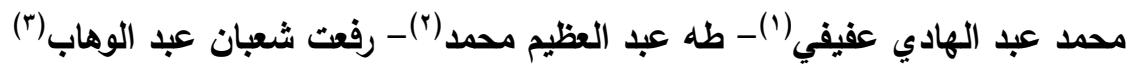

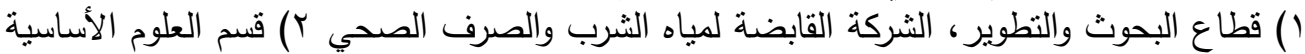

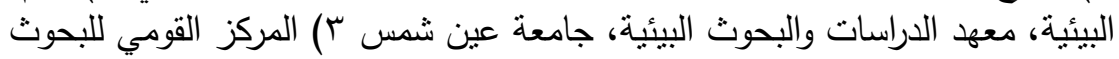

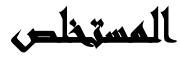

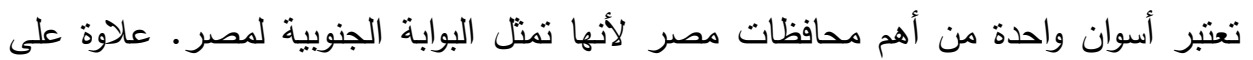

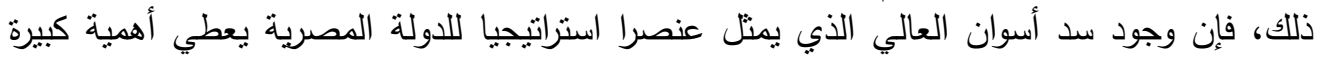

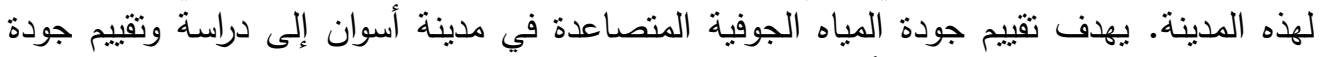

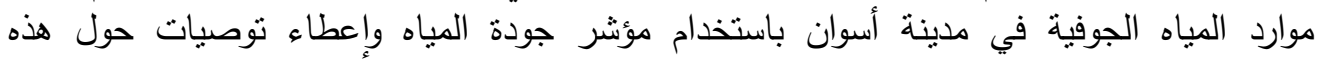

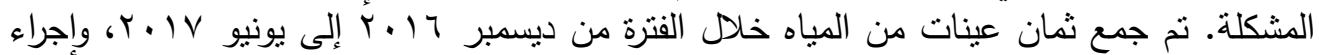

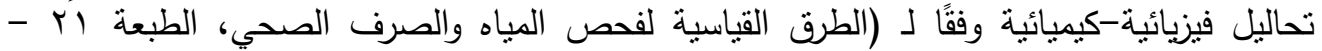

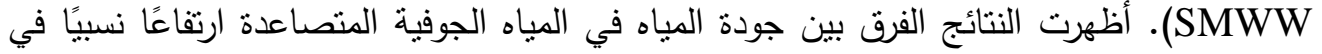

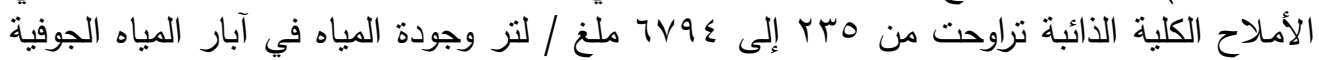

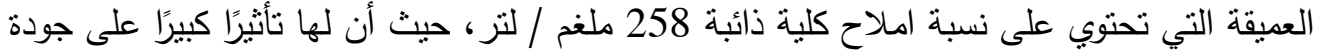

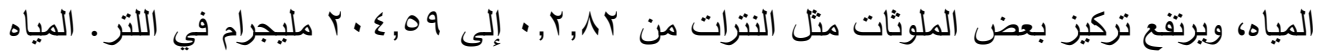

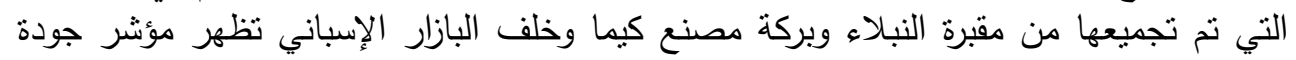
71.2

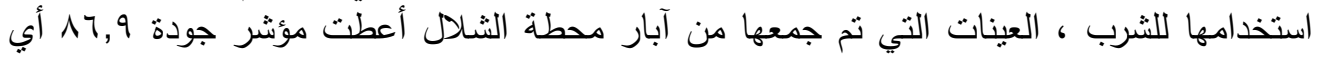

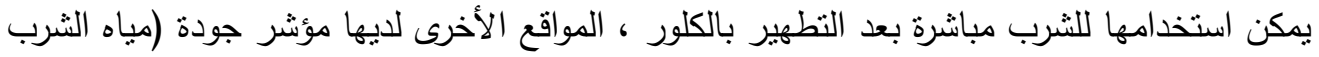

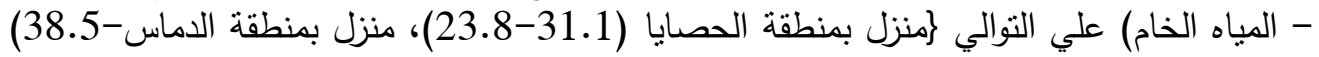

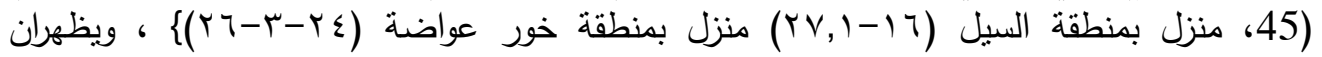

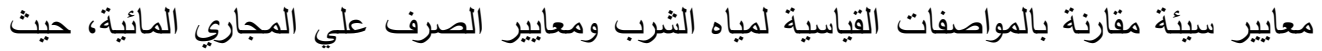

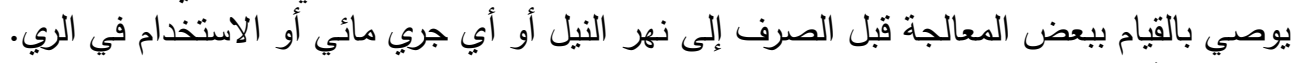

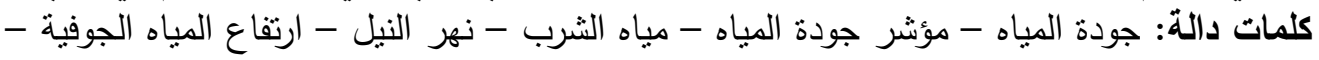
الخزان الجوفي - الآبار العميقة. دؤن 